\title{
Breast-feeding duration for the prevention of excess body weight of mother-child pairs concurrently: a 2-year cohort study
}

\author{
Marco Fabio Mastroeni ${ }^{1,2, *}$, Silmara Salete de Barros Silva Mastroeni ${ }^{1,3}$, \\ Sandra Ana Czarnobay ${ }^{2}$, John Paul Ekwaru', Sarah A Loehr ${ }^{1}$ and Paul J Veugelers ${ }^{1}$ \\ 'Population Health Intervention Research Unit, School of Public Health, University of Alberta, Edmonton, Alberta, \\ Canada: ${ }^{2}$ Postgraduate Program in Health and Environment, University of Joinville Region, Rua Paulo Malschitzki no. \\ 10, Joinville, Santa Catarina, CEP 89.219-710, Brazil: ${ }^{3}$ Department of Physical Education, University of Joinville \\ Region, Joinville, Santa Catarina, Brazil
}

Submitted 9 September 2016: Final revision received 4 May 2017: Accepted 16 May 2017: First published online 19 June 2017

\begin{abstract}
Objective: To examine the association between breast-feeding duration and the risk of excess body weight (children $>85$ th percentile, mothers BMI $\geq 25 \cdot 0 \mathrm{~kg} / \mathrm{m}^{2}$ ) concurrently in mother-child pairs two years after delivery.

Design: Prospective cohort study in Joinville, Brazil. Multivariable logistic regression was used to examine the independent relationship between breastfeeding duration and risk of excess body weight.

Setting: Brazilian public maternity hospital.

Subjects: Three hundred and five mother-child pairs.

Results: At 2-year follow-up, $23.6 \%$ of mother-child pairs had excess body weight. Children breast-fed for $<2$ months were more likely to have excess body weight than children breast-fed for $\geq 6$ months (OR $=2 \cdot 4 ; 95 \%$ CI 1·1, 5·1). Breast-feeding for $<2$ months was also associated with a greater likelihood of maternal excess body weight compared with those who breast-fed for $\geq 6$ months (OR $=2.9 ; 95 \%$ CI $1 \cdot 1,8 \cdot 1)$. There was a progressive increase in the likelihood of mother-child pairs having excess body weight as breast-feeding duration decreased. In addition to breast-feeding duration, other independent determinants of excess body weight were pre-pregnancy weight, gestational weight gain and number of pregnancies in mothers, and birth weight in children.

Conclusions: Breast-feeding for a longer duration has a parallel protective effect on the risk of excess body weight in mother-child pairs two years after birth. Since members of the same family could be influenced by the same risk factors, continued promotion and support of breast-feeding may help to attenuate the rising prevalence of overweight in mother-child pairs.
\end{abstract}

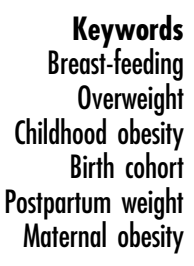

The worldwide prevalence of overweight and obesity in adults and children has increased substantially in the last 30 years and, as such, has become a major global health challenge $^{(1)}$. In Brazil from 1975 and 2009, the prevalence of overweight and obesity in adult women increased from 28.7 to $48.0 \%$ and from 8.0 to $16.9 \%$, respectively ${ }^{(2)}$. Over the same time period, the prevalence of obesity in Brazilian children aged 5-9 years increased from 2.4 to $14 \cdot 2 \%{ }^{(2)}$.

The postpartum period has been identified as a time of increased vulnerability for maternal weight gain ${ }^{(3)}$. Failure to lose weight after pregnancy is an important predictor of long-term obesity, increasing a mother's risk for CVD, hypertension, diabetes and degenerative joint disease ${ }^{(4,5)}$. This time period may also be crucial for the long-term weight status of the child, as increasing evidence has emerged showing that both excessive weight gain and fast weight gain in early childhood are associated with excess body weight later in life ${ }^{(6-8)}$. Thus, the postpartum period and early infancy provide an important public health opportunity for the primary prevention of obesity for both mother and child $^{(9)}$.

It has been proposed that maternal lactation and early feeding practices decrease both maternal postpartum weight retention and the development of obesity in childhood. However, evidence that breast-feeding duration has a protective effect for either mother or child has been equivocal. Several longitudinal studies have shown that children who were breast-fed for a shorter duration during infancy were less satiety responsive ${ }^{(10)}$, had more 
unhealthy snacking behaviours ${ }^{(11)}$ and were at higher risk of being overweight/obese ${ }^{(11-24)}$. Additionally, longitudinal studies conducted in Sweden, Germany, Australia, the USA, Denmark and Brazil have reported that breastfeeding for a longer duration was associated with a decreased risk of maternal postpartum excess body weight $^{(1,18,22,25-28)}$. Other studies, however, have reported small or no protective effects of breast-feeding duration on obesity in children ${ }^{(10,29-38)}$ or in women after delivery ${ }^{(37)}$.

A causal link between breast-feeding duration and childhood obesity has been difficult to establish ${ }^{(39)}$ mainly because of confounding maternal, child, cultural, genetic and environmental variables ${ }^{(40)}$. In addition, most studies performed to date have been cross-sectional. Only three prospective cohort studies have evaluated the association between breast-feeding duration and excess body weight individually in mothers and their children ${ }^{(18,22,41)}$, and no cohort study, to our knowledge, has performed such analysis concurrently in mother-child pairs for two years. This is important from a public health perspective since members of the same family are influenced by several of the same risk factors, some of which may be modified with pre- and postnatal care. Therefore, in the present study, we investigated the relationship between breast-feeding duration and risk of excess body weight in Brazilian women in concert with their children two years after delivery.

\section{Methods}

\section{Study design and population}

Data came from the Predictors of Maternal and Infant Excess Body Weight (PREDI) Study. Recruitment for this prospective cohort study took place between 14 January and 16 February 2012 at the public maternity hospital in Joinville, a city of about 500000 inhabitants in Santa Catarina, Brazil. Details of the recruitment process have been previously described ${ }^{(42,43)}$. Briefly, all women over the age of 18 years, who gave birth to a full-term singleton (between 37 and 42 weeks of gestation), were invited to participate with their newborn infants in the first stage of the study (first investigation). Exclusion criteria included pre-eclampsia, the presence of an infectious contagious disease (AIDS, hepatitis, syphilis and toxoplasmosis), birth defects and plans for adoption immediately after delivery. Of the 529 eligible pairs (mothers and infants), fifty-eight did not meet the study criteria and thirty-six were not considered for other reasons, resulting in a total of 435 mother-infant pairs who participated in the first investigation (Fig. 1).

The second stage of the study (second investigation) was carried out in the homes of the participants between March 2013 and March 2014. All mothers and children who participated in the first stage of the study were invited to participate in the study's second stage. Children with an

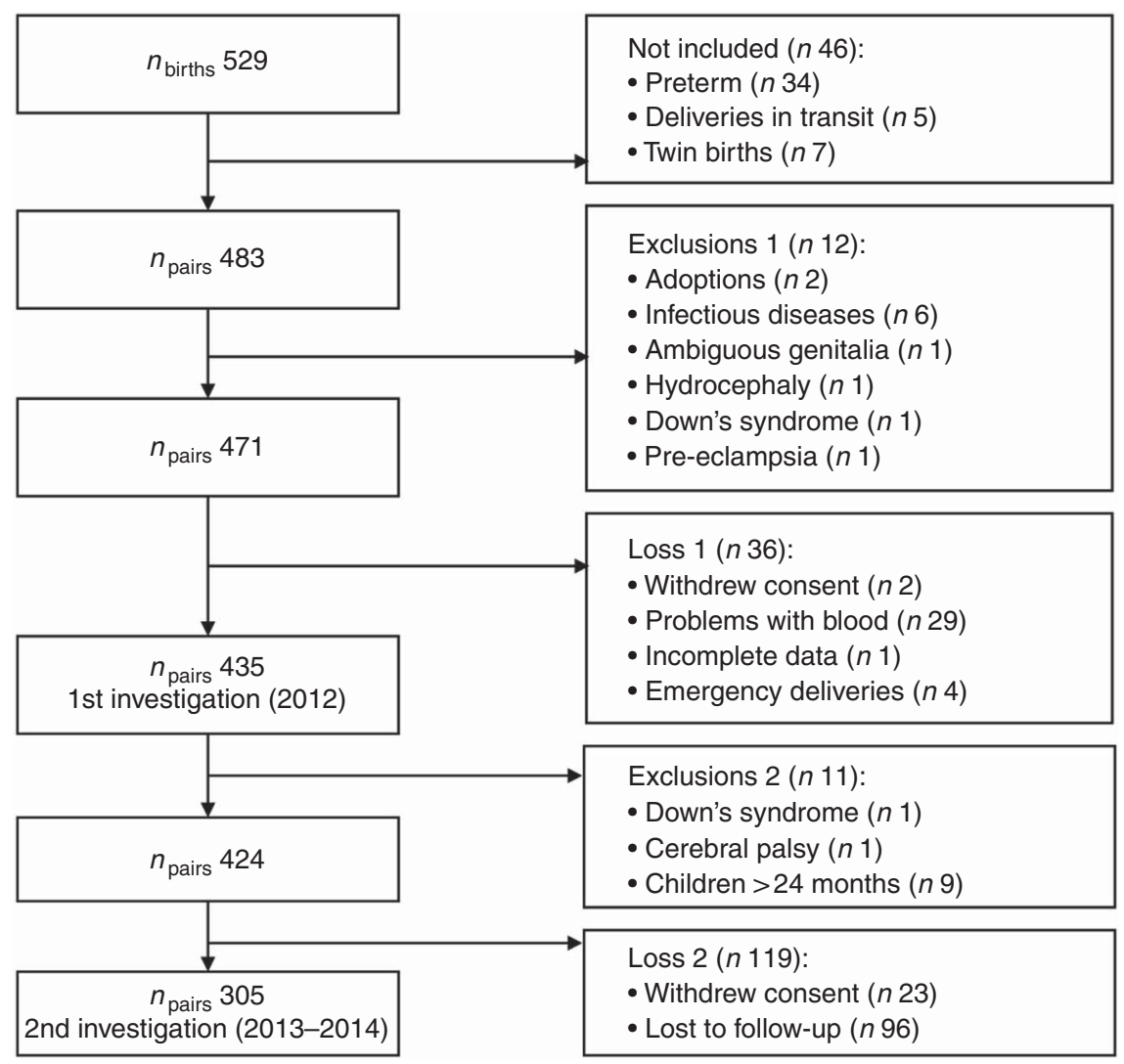

Fig. 1 Flow chart of participants through the recruitment process, Joinville, Brazil 
anomaly that interfered with anthropometric measurements were excluded from the second investigation. Of the 435 pairs who participated in the first investigation, eleven did not meet the inclusion criteria for the second investigation and 119 were considered lost to follow-up, resulting in $305(70 \cdot 1 \%)$ mother-child pairs (Fig. 1).

\section{Data collection}

First investigation

Within $48 \mathrm{~h}$ after the birth of their infant, mothers received information about the study. If both mother and infant met the study's inclusion criteria, they were invited to participate by providing informed consent.

Once enrolled in the study, mothers completed a previously tested structured questionnaire to collect demographic, socio-economic, anthropometric, obstetric and reproductive information on themselves and their newborns. Details of the data collection tools have been previously described $^{(42,43)}$. The questionnaire was administered by trained health professionals in a private maternity hospital room within $48 \mathrm{~h}$ after delivery, at the time we assessed their postpartum height. Postpartum height was measured to the nearest $0.1 \mathrm{~cm}$ using a portable stadiometer $\left(\mathrm{WCS}^{\circledR}, \mathrm{Com}-\right.$ pact model) on a wall without skirting. The mothers stood in an orthostatic position, wearing light clothing with no shoes and having their weight evenly distributed. Pre-pregnancy BMI ([weight $(\mathrm{kg})] /$ height $(\mathrm{m})]^{2}$ ) was calculated based on the mothers' self-reported pre-pregnancy weight and postpartum measured height. Pre-pregnancy weight was classified according to the WHO BMI cut-offs ${ }^{(44)}$, which classify individuals with a $\mathrm{BMI}<18.5 \mathrm{~kg} / \mathrm{m}^{2}$ as being underweight, BMI between 18.5 and $24.9 \mathrm{~kg} / \mathrm{m}^{2}$ as being normal weight, BMI between 25.0 and $29.9 \mathrm{~kg} / \mathrm{m}^{2}$ as being overweight, and $\mathrm{BMI} \geq 30 \cdot 0 \mathrm{~kg} / \mathrm{m}^{2}$ as being obese. We combined underweight ( $n$ 19) and normal weight ( $n$ 251) mothers into one category (pre-pregnancy BMI $<25 \cdot 0 \mathrm{~kg} / \mathrm{m}^{2}$ ).

Gestational weight gain (GWG) was obtained by subtracting the pre-pregnancy weight from the weight at delivery (measured on the day of delivery). The adequacy of GWG was assessed according to the 2009 Institute of Medicine guidelines, which state that underweight women should gain between 12.5 and $18.0 \mathrm{~kg}$, women with adequate BMI between 11.5 and $16.0 \mathrm{~kg}$, overweight women between $7 \cdot 0$ and $11.5 \mathrm{~kg}$, and obese women between $5 \cdot 0$ and $9.0 \mathrm{~kg}^{(45)}$.

Birth weight and length of the infants were collected from hospital records. Birth weight was classified into three categories according to gestational age and sex: small for gestational age (SGA; <10th percentile), adequate for gestational age (AGA; 10-90th percentile) and large for gestational age (LGA; >90th percentile) ${ }^{(46)}$.

\section{Second investigation}

Trained personnel completed the second investigation data collection at the homes of the study participants.
Anthropometric measurements were performed on both mother and child and demographic and socio-economic information was collected individually using a previously tested structured questionnaire in a private room of the family's home. Children's weight was measured to the nearest $10 \mathrm{~g}$ using a paediatric digital portable scale (Beurer $^{\circledR}$, BY20 model) and length was measured to the nearest $0 \cdot 1 \mathrm{~cm}$ using a paediatric anthropometric ruler (WCS ${ }^{\circledR}$, Wood model).

The children's weight status was divided into two categories based on the 2006 WHO growth standards for weight-for-age: $>85$ th percentile and $\leq 85$ th percentile ${ }^{(47)}$. The mothers' height was measured using the same equipment and procedures as employed in the first investigation. Their weight was measured on a digital scale (Plenna $^{\circledR}$, Acqua model) with a capacity of $180 \mathrm{~kg}$ to the nearest $0 \cdot 1 \mathrm{~kg}$ while wearing light clothes without shoes and accessories (jewellery, watches, coats). All anthropometric measures were performed in duplicate in both the first and second investigations. The mean of the two measurements was used for analysis. Maternal weight status was classified using the same WHO BMI cut-offs ${ }^{(44)}$ as employed in the first investigation for pre-pregnancy weight.

Breast-feeding duration (in months) was self-reported by the mothers. Breast-feeding was classified according to the WHO indicators for assessing infant and young child feeding practices, which define 'exclusive breast-feeding' when the infant receives only breast milk or expressed milk and no other liquid or solid (except for drops or syrups of vitamins, minerals and/or medications) for a period of 6 months or more; 'predominant breast-feeding' when the infant receives breast milk or expressed milk, as well as water and water-based drinks such as fruit juice and tea; 'complementary feeding' when the infant receives breast milk or expressed milk, as well as solid or semisolid foods, non-human milk and special formula; 'breastfeeding' when the infant receives breast milk or expressed milk, as well as non-human milk and special formula; and 'artificial feeding' when the infant receives any type of liquid or semi-solid food in a bottle, including breast milk, non-human milk and special formula ${ }^{(48)}$. For the current study, all non-exclusive breast-feeding categories were grouped into a single non-exclusive breast-feeding category $^{(48)}$.

\section{Statistical analyses}

Data were analysed using the statistical software package IBM SPSS Statistics version 22.0. To examine the differences between the mother-child pairs who participated in the second investigation ( $n$ 305) and those who did not participate ( $n 130$ from the first investigation), we used the $\chi^{2}$ test to compare the variables age, education, birth weight and sex. The $\chi^{2}$ test was also used to compare the prevalence of categorical variables according to weight status of the children ( $>85$ th percentile and $\leq 85$ th 
percentile) and the mothers (underweight/normal weight, overweight and obese). Mother's age $(<20,20-30$ and $\geq 30$ years old) and monthly household income $(<3,3-5$ and $\geq 5$ minimum salaries) were classified according to the Brazilian Institute of Geography and Statistics' criteria ${ }^{(49)}$. For mother's age we grouped the categories of 20-24 years and 25-29 years into a single category (20-30 years). Mother's education ( $<9$ years for the mothers who completed primary school; 9-12 years for those who completed high school; $\geq 12$ years for those who started/ finished undergraduate courses) was classified according to the 1996 Education Law (Lei das Diretrizes e Bases da Educação Nacional) ${ }^{(50)}$.

To investigate the association of children with a weight status $>85$ th percentile and mothers with a BMI $\geq 25.0 \mathrm{~kg} / \mathrm{m}^{2}$ with breast-feeding duration and other risk factors, the OR and $95 \% \mathrm{CI}$ were calculated using logistic regression. Unadjusted analyses (Models 1, 3 and 5) were used to estimate the crude association of each exposure with the outcome for children with a weight status $>85$ th percentile, mothers with a BMI $\geq 25.0 \mathrm{~kg} / \mathrm{m}^{2}$ and mother-child pairs concurrently with excess body weight (children with a weight status $>85$ th percentile and mothers with a BMI $\geq 25 \cdot 0 \mathrm{~kg} / \mathrm{m}^{2}$ ), relative to children with a weight status $\leq 85$ th percentile, mothers with a BMI $<25.0 \mathrm{~kg} / \mathrm{m}^{2}$ and mother-child pairs without excess body weight, respectively. The covariates breast-feeding duration, months since delivery, sex, birth weight, type of delivery, mother's age, mother's education, marital status, monthly household income, smoking after pregnancy, pre-pregnancy BMI, GWG and number of pregnancies were simultaneously considered in multivariable logistic regression models (Models 2, 4 and 6) to reveal the independent importance of breast-feeding duration on excess body weight. These variables have been shown in the literature to be related with both breast-feeding duration and the outcome of interest. We performed the variance inflator factor test with commonly applied cut-off values to reveal little collinearity among the independent variables ${ }^{(51)}$. All analyses were considered statistically significant when $P<0 \cdot 05$.

\section{Results}

The $\chi^{2}$ tests for proportionality showed no significant difference in age, education, birth weight and sex between the mother-child pairs enrolled in the second investigation ( $n$ 305) and those enrolled in the first investigation only ( $n$ 435). The characteristics of the mothers and children grouped by weight status are presented in Table 1 . Of the 305 mothers and children included in the study, 126 (41.6\%) children had a body weight $>85$ th percentile. A larger proportion of children who were breast-fed for $<2$ months (56.9\%) or who were LGA at birth (56.8\%) had a body weight $>85$ th percentile at 2 years of age compared with children who were breast-fed for longer or who were SGA or AGA at birth. At 2-year follow-up, 146 (48.8\%) mothers were either overweight or obese (BMI $\geq 25 \cdot 0$ $\mathrm{kg} / \mathrm{m}^{2}$ ). Compared with those who were underweight/ normal weight, a greater proportion of overweight and obese mothers had caesarean sections (53.6\%), were overweight/obese prior to pregnancy (90.4\%), had excessive GWG (68.1\%) and a greater number of pregnancies (59.6\%; Table 1).

Table 2 presents the characteristics of mother-child pairs by weight status two years after delivery. Regarding mother-child pairs, $71(23.6 \%)$ were found to have excess body weight in both mother and child (children $>85$ th percentile and mothers BMI $\geq 25 \cdot 0 \mathrm{~kg} / \mathrm{m}^{2}$ ) at 2 -year follow-up. There was a significant increase in the proportion of mother-child pairs with excess body weight in the mothers who had excessive GWG (13.5 to $35.5 \%$, $P<0.01)$ and had breast-fed for less time (18.0 to $36.0 \%$, $P=0 \cdot 02)$.

Table 3 presents the determinants of excess body weight in children, mothers and mother-child pairs at 2-year follow-up. Unadjusted analyses (Model 1) showed that as breast-feeding duration decreased the likelihood of children having excess body weight progressively increased $(\mathrm{OR}=1 \cdot 3 ; 95 \% \mathrm{CI} 0 \cdot 7,2 \cdot 3 ; P=0 \cdot 40, \mathrm{OR}=2 \cdot 0$; $95 \%$ CI $1 \cdot 0,4 \cdot 1 ; P=0.04$ and $\mathrm{OR}=2 \cdot 7 ; 95 \%$ CI $1 \cdot 5,7 \cdot 0$; $P<0 \cdot 01$, for $4-<6,2-<4$ and $<2$ months, respectively). Children who were LGA at birth or who had mothers who gained excessive weight during pregnancy also had a significantly greater likelihood of having excess body weight at follow-up $(\mathrm{OR}=2.4 ; 95 \%$ CI 1.4, 4.0; $P<0.01$ and $\mathrm{OR}=1.6 ; 95 \% \mathrm{CI} 1.0,2.5 ; P<0 \cdot 04$, respectively). These results did not change substantially after adjusting for important potential confounding variables (Model 2). Children who were breast-fed for $<2$ months were 2.4 (95\% CI $1 \cdot 1,5 \cdot 1 ; P=0.02)$ times as likely to have excess body weight compared with children who were breast-fed for $\geq 6$ months (Model 2). Children who were LGA at birth and those whose monthly household income was $<3$ minimum salaries also had a significantly greater likelihood of having excess body weight compared with SGA/AGA children and those with a monthly household income $\geq 3$ minimum salaries $(\mathrm{OR}=2 \cdot 7 ; 95 \%$ CI $1.5,4.9 ; P<0.01$ and $\mathrm{OR}=1 \cdot 8 ; 95 \%$ CI $1 \cdot 0,3 \cdot 1 ; P=0 \cdot 03$, respectively).

Regarding the mothers, unadjusted analyses in Table 3 revealed that breast-feeding duration, time since delivery, pre-pregnancy BMI, excessive GWG and number of pregnancies were significantly associated with an increased risk of being overweight/obese at follow-up (Model 3). After adjusting for potential confounders, breast-feeding duration, pre-pregnancy BMI, GWG and number of pregnancies showed independent effects on excess body weight (Model 4). Mothers who breast-fed for $<2$ months were 2.9 (95\% CI $1 \cdot 1,8 \cdot 1 ; P=0.04)$ times as likely to be overweight/obese when compared with those who breast-fed for $\geq 6$ months (Model 4). 
Table 1 Characteristics of mothers and their children by weight status two years after delivery $(n \text { 301 })^{\star}$, Joinville, Brazil 2013-2014

\begin{tabular}{|c|c|c|c|c|c|c|c|c|c|c|c|c|c|c|c|c|}
\hline \multirow[b]{3}{*}{ Characteristic } & \multicolumn{7}{|c|}{ Children's weight status } & \multicolumn{9}{|c|}{ Mother's weight status } \\
\hline & \multicolumn{2}{|c|}{$\begin{array}{l}\leq 85 \text { th percentile } \\
\quad(58.7 \%)\end{array}$} & \multicolumn{2}{|c|}{$\begin{array}{l}>85 \text { th percentile } \\
\quad(41.3 \%)\end{array}$} & \multicolumn{2}{|c|}{$\begin{array}{c}\text { Total } \\
(100 \cdot 0 \%)\end{array}$} & \multirow[b]{2}{*}{$P$ value } & \multicolumn{2}{|c|}{$\begin{array}{l}\text { Underweight/normal weight } \\
\qquad(51.2 \%)\end{array}$} & \multicolumn{2}{|c|}{$\begin{array}{l}\text { Overweight } \\
(28.6 \%)\end{array}$} & \multicolumn{2}{|c|}{$\begin{array}{l}\text { Obese } \\
(20.2 \%)\end{array}$} & \multicolumn{2}{|c|}{$\begin{array}{c}\text { Total } \\
(100 \cdot 0 \%)\end{array}$} & \multirow[b]{2}{*}{$P$ value } \\
\hline & $n$ & $\%$ & $n$ & $\%$ & $n$ & $\%$ & & $n$ & $\%$ & $n$ & $\%$ & $n$ & $\%$ & $n$ & $\%$ & \\
\hline \multicolumn{17}{|l|}{ Children } \\
\hline Breast-feeding duration (months) $\dagger$ & & & & & & & 0.02 & & & & & & & & & \multirow[t]{5}{*}{0.18} \\
\hline o sa & 69 & $67 \cdot 0$ & 34 & 33.0 & 103 & $34 \cdot 0$ & & 60 & $60 \cdot 0$ & 20 & $20 \cdot 0$ & 20 & $20 \cdot 0$ & 100 & $33 \cdot 4$ & \\
\hline $4-<6$ & 62 & $61 \cdot 4$ & 39 & 38.6 & 101 & 33.3 & & 48 & 47.5 & 32 & 31.7 & 21 & 20.8 & 101 & 33.8 & \\
\hline $2-<4$ & 24 & $50 \cdot 0$ & 24 & 50.0 & 48 & $15 \cdot 8$ & & 20 & 41.7 & 15 & $31 \cdot 2$ & 13 & $27 \cdot 1$ & 48 & $16 \cdot 1$ & \\
\hline$<2$ & 22 & 43.1 & 29 & 56.9 & 51 & $16 \cdot 9$ & & 25 & $50 \cdot 0$ & 18 & 36.0 & 7 & 14.0 & 50 & $16 \cdot 7$ & \\
\hline \multicolumn{7}{|l|}{ Type of breast-feeding $\dagger$} & \multirow[t]{3}{*}{0.04} & & & & & & & & & \multirow[t]{3}{*}{0.12} \\
\hline Exclusive & 70 & $66 \cdot 0$ & 36 & 34.0 & 106 & $35 \cdot 0$ & & 61 & $59 \cdot 2$ & 21 & $20 \cdot 8$ & 21 & $20 \cdot 4$ & 103 & 34.4 & \\
\hline Non exclusive & 107 & $54 \cdot 3$ & 90 & $45 \cdot 7$ & 197 & $65 \cdot 0$ & & 92 & 47.0 & 64 & 32.6 & 40 & $20 \cdot 4$ & 196 & 65.6 & \\
\hline \multicolumn{7}{|l|}{ Time since delivery (months) } & 0.07 & & & & & & & & & 0.13 \\
\hline$<18$ & 123 & $62 \cdot 4$ & 74 & 37.6 & 197 & 65.5 & & 107 & $55 \cdot 4$ & 51 & $26 \cdot 5$ & 35 & $18 \cdot 1$ & 193 & $64 \cdot 1$ & \\
\hline$\geq 18$ & 56 & $51 \cdot 8$ & 52 & $48 \cdot 1$ & 108 & 34.5 & & 47 & 43.5 & 35 & 32.4 & 26 & $24 \cdot 1$ & 108 & $35 \cdot 9$ & \\
\hline Sex & & & & & & & 0.82 & & & & & & & & & 0.48 \\
\hline Male & 90 & 58.0 & 65 & $42 \cdot 0$ & 155 & $50 \cdot 8$ & & 77 & $50 \cdot 3$ & 41 & $26 \cdot 8$ & 35 & $22 \cdot 9$ & 153 & $50 \cdot 8$ & \\
\hline Female & 89 & $59 \cdot 3$ & 61 & 40.7 & 150 & 49.2 & & 77 & $52 \cdot 0$ & 45 & 30.4 & 26 & $17 \cdot 6$ & 148 & $49 \cdot 2$ & \\
\hline Birth weight & & & & & & & $<0.01$ & & & & & & & & & 0.15 \\
\hline SGA/AGA & 144 & $64 \cdot 2$ & 80 & $35 \cdot 8$ & 224 & 73.4 & & 120 & $54 \cdot 3$ & 61 & $27 \cdot 6$ & 40 & $18 \cdot 1$ & 221 & 73.4 & \\
\hline LGA & 35 & 43.2 & 46 & $56 \cdot 8$ & 81 & $26 \cdot 6$ & & 34 & 42.5 & 25 & 31.3 & 21 & $26 \cdot 2$ & 80 & $26 \cdot 6$ & \\
\hline Type of delivery & & & & & & & 0.53 & & & & & & & & & 0.03 \\
\hline Normal & 124 & $60 \cdot 0$ & 83 & $40 \cdot 0$ & 207 & $67 \cdot 8$ & & 109 & 53.4 & 62 & 30.4 & 33 & $16 \cdot 2$ & 204 & $67 \cdot 8$ & \\
\hline Caesarean section & 55 & $56 \cdot 1$ & 43 & 43.9 & 98 & $32 \cdot 2$ & & 45 & $46 \cdot 4$ & 24 & $24 \cdot 7$ & 28 & $28 \cdot 9$ & 97 & $32 \cdot 2$ & \\
\hline Mothers & & & & & & & & & & & & & & & & \\
\hline Age (years) & & & & & & & 0.37 & & & & & & & & & 0.14 \\
\hline $20-30$ & 109 & $57 \cdot 7$ & 80 & $42 \cdot 3$ & 189 & $62 \cdot 0$ & & 5 & $55 \cdot 6$ & 1 & $11 \cdot 1$ & 3 & $33 \cdot 3$ & 9 & 3.0 & \\
\hline$<20$ & 8 & $80 \cdot 0$ & 2 & $20 \cdot 0$ & 10 & $3 \cdot 3$ & & 102 & $54 \cdot 8$ & 54 & 29.0 & 30 & $16 \cdot 1$ & 186 & 61.8 & \\
\hline$\geq 30$ & 62 & 58.5 & $4 \overline{4}$ & 41.5 & 106 & 34.7 & & 47 & $44 \cdot 3$ & 31 & 29.4 & 28 & $26 \cdot 4$ & 106 & $35 \cdot 2$ & \\
\hline Education (years) & & & & & & & 0.58 & & & & & & & & & 0.16 \\
\hline$\geq 12$ & 42 & 63.3 & 24 & 36.7 & 66 & 21.6 & & 34 & $52 \cdot 3$ & 20 & 30.7 & 11 & $16 \cdot 9$ & 65 & 21.6 & \\
\hline $9-12$ & 82 & $56 \cdot 1$ & 64 & 43.9 & 146 & 47.9 & & 81 & $56 \cdot 2$ & 39 & $27 \cdot 1$ & 24 & $16 \cdot 7$ & 144 & 47.8 & \\
\hline$<9$ & 55 & $59 \cdot 1$ & 38 & 40.8 & 93 & 30.5 & & 39 & $42 \cdot 4$ & 27 & $29 \cdot 3$ & 26 & $28 \cdot 3$ & 92 & 30.6 & \\
\hline Marital status & & & & & & & 0.89 & & & & & & & & & 0.34 \\
\hline Marriage/consensual union & 160 & 58.8 & 112 & $41 \cdot 2$ & 272 & $89 \cdot 2$ & & 134 & $49 \cdot 8$ & 80 & 29.7 & 55 & $20 \cdot 0$ & 269 & 89.4 & \\
\hline Others & 19 & 57.6 & 14 & $42 \cdot 4$ & 33 & $10 \cdot 8$ & & 20 & 62.6 & 6 & $18 \cdot 7$ & 6 & 18.7 & 32 & $10 \cdot 6$ & \\
\hline Monthly household income (MS)‡ & & & & & & & 0.26 & & & & & & & & & 0.05 \\
\hline$\geq 5$ & 36 & 65.4 & 19 & 34.5 & 55 & $18 \cdot 2$ & & 87 & $50 \cdot 0$ & 43 & 24.7 & 44 & $25 \cdot 3$ & 174 & 58.4 & \\
\hline $3-5$ & 44 & $62 \cdot 8$ & 26 & $37 \cdot 2$ & 70 & 23.2 & & 41 & 58.6 & 20 & $28 \cdot 6$ & 9 & $12 \cdot 8$ & 70 & 23.5 & \\
\hline$<3$ & 97 & $54 \cdot 8$ & 80 & $45 \cdot 2$ & 177 & 58.6 & & 26 & 48.1 & 21 & 38.9 & 7 & 12.9 & 54 & $18 \cdot 1$ & \\
\hline Working/studying after delivery† & & & & & & & 0.07 & & & & & & & & & 0.16 \\
\hline No & 78 & $65 \cdot 0$ & 42 & $35 \cdot 0$ & 120 & 39.6 & & 55 & $46 \cdot 6$ & 33 & 28.0 & 30 & $25 \cdot 4$ & 118 & 39.5 & \\
\hline Yes & 100 & $54 \cdot 6$ & 83 & $45 \cdot 4$ & 183 & $60 \cdot 4$ & & 98 & $54 \cdot 1$ & 53 & $29 \cdot 3$ & 30 & $16 \cdot 6$ & 181 & 60.5 & \\
\hline Smoking after pregnancy & & & & & & & 0.46 & & & & & & & & & 0.27 \\
\hline No & 154 & $57 \cdot 9$ & 112 & $42 \cdot 1$ & 266 & $87 \cdot 2$ & & 137 & $52 \cdot 1$ & 71 & $27 \cdot 0$ & 55 & $20 \cdot 9$ & 263 & $87 \cdot 4$ & \\
\hline Yes & 25 & $64 \cdot 1$ & 14 & 35.9 & 39 & $12 \cdot 8$ & & 17 & $44 \cdot 7$ & 15 & 39.5 & 6 & $15 \cdot 8$ & 38 & 12.6 & \\
\hline
\end{tabular}


Models 5 and 6 in Table 3 present data from the analyses of mother-child pairs who were both categorized as having excess body weight. Breast-feeding duration, time since delivery, child's birth weight, maternal prepregnancy BMI and GWG were significantly associated with an increased risk of excess body weight concurrently on mother-child pairs (Model 5). Except for time since delivery, these associations continued to be significant after adjusting for confounders (Model 6). Furthermore, there was a progressive increase in the likelihood of concurrent excess body weight in mother-child pairs as breast-feeding duration decreased (Model 6: $\mathrm{OR}=0.9$; $95 \%$ CI $0.4,2 \cdot 0 ; P=0.71, \quad$ OR $=1 \cdot 7 ; 95 \%$ CI $0 \cdot 6,4 \cdot 3$; $P=0 \cdot 30$ and $\mathrm{OR}=3 \cdot 2 ; 95 \% \mathrm{CI} 1 \cdot 3,8 \cdot 2 ; P=0.01$ for $4-<6$, $2-<4$ and $<2$ months, respectively).

The risk of excess body weight in mothers and their children according to breast-feeding duration is depicted in Fig. 2. Estimates were adjusted for time since delivery, sex, birth weight, type of delivery, mother's age, mother's education, marital status, household income, smoking after pregnancy, pre-pregnancy BMI, GWG and number of pregnancies. The reference group was considered those who breast-fed for $\geq 6$ months. Mothers and children who partook in breast-feeding for $<2$ months had the greatest risk of excess body weight at follow-up, with mothers having a greater risk than children. The greatest risk was observed when analysis was performed concurrently on mother-child pairs.

\section{Discussion}

In the current investigation, we found that the risk of a mother and her child having excess body weight progressively increases as breast-feeding duration decreases. Furthermore, our findings indicated that longer breast-feeding duration is an independent contributor to reducing the risk of excess body weight in children and mothers individually, as well as concurrently in both the mother and her child, even when other possible confounding factors such as birth weight, pre-pregnancy BMI and GWG are considered.

Although this protective effect has been reported in several other studies ${ }^{(1,11-28)}$, many longitudinal studies have produced conflicting results regarding the benefits of breast-feeding duration on the prevention of excess body weight in mothers and children. The majority of these longitudinal prospective studies, however, did not investigate both mothers and children concurrently, but rather either only the mothers or only the children $^{(11-17,19-21,23,28,37,52)}$. The current study design in which analyses of mother-child pairs was concurrently performed is important from a public health perspective since members of the same family could be influenced by several of the same risk factors, some of which may be modified with pre- and postnatal care. Increasing our understanding on how breast-feeding duration may impact 
Table 2 Characteristics of mother-child pairs by weight status two years after delivery ( $n$ 301), Joinville, Brazil, 2013-2014

\begin{tabular}{|c|c|c|c|c|c|c|c|}
\hline \multirow[b]{3}{*}{ Characteristic } & \multicolumn{4}{|c|}{ Weight status (BMI) } & \multirow{2}{*}{\multicolumn{2}{|c|}{$\begin{array}{c}\text { Total } \\
(100.0 \%)\end{array}$}} & \multirow[b]{3}{*}{$P$ value } \\
\hline & \multicolumn{2}{|c|}{$\begin{array}{c}\text { Children } \leq 85 \text { th percentile, } \\
\text { mothers } \mathrm{BMl}<25 \mathrm{~kg} / \mathrm{m}^{2} \\
(76.4 \%)\end{array}$} & \multicolumn{2}{|c|}{$\begin{array}{c}\text { Children }>85 \text { th percentile, } \\
\text { mothers } \mathrm{BMl} \geq 25 \mathrm{~kg} / \mathrm{m}^{2} \\
(23.6 \%)\end{array}$} & & & \\
\hline & $n$ & $\%$ & $n$ & $\%$ & $n$ & $\%$ & \\
\hline \multicolumn{8}{|l|}{ Children } \\
\hline \multicolumn{7}{|l|}{ Breast-feeding duration (months)* } & 0.02 \\
\hline$\geq 6$ & 82 & $82 \cdot 0$ & 18 & $18 \cdot 0$ & 100 & 33.5 & \\
\hline $4-<6$ & 82 & $81 \cdot 2$ & 19 & 18.8 & 101 & 33.8 & \\
\hline $2-<4$ & 32 & $66 \cdot 7$ & 16 & 33.3 & 48 & $16 \cdot 0$ & \\
\hline$<2$ & 32 & $64 \cdot 0$ & 18 & $36 \cdot 0$ & 50 & $16 \cdot 7$ & \\
\hline \multicolumn{7}{|l|}{ Type of breast-feeding ${ }^{*}$} & 0.11 \\
\hline Exclusive & 84 & 81.6 & 19 & 18.4 & 103 & 34.4 & \\
\hline Non exclusive & 144 & 73.5 & 52 & 26.5 & 196 & $65 \cdot 6$ & \\
\hline \multicolumn{7}{|l|}{ Time since delivery (months) } & 0.03 \\
\hline$<18$ & 155 & $80 \cdot 3$ & 38 & 19.7 & 193 & $64 \cdot 1$ & \\
\hline$\geq 18$ & 75 & 69.4 & 33 & 30.6 & 108 & $35 \cdot 9$ & \\
\hline \multicolumn{7}{|l|}{$\operatorname{sex}$} & 0.98 \\
\hline Male & 117 & 76.5 & 36 & 23.5 & 153 & $50 \cdot 8$ & \\
\hline Female & 113 & $76 \cdot 4$ & 35 & $23 \cdot 6$ & 148 & $49 \cdot 2$ & \\
\hline \multicolumn{7}{|l|}{ Birth weight } & $<0.01$ \\
\hline SGA/AGA & 181 & 81.9 & 40 & $18 \cdot 1$ & 221 & 73.4 & \\
\hline LGA & 49 & $61 \cdot 2$ & 31 & $38 \cdot 8$ & 80 & $26 \cdot 6$ & \\
\hline \multicolumn{7}{|l|}{ Type of delivery } & 0.23 \\
\hline Normal & 160 & 78.4 & 44 & 21.6 & 204 & $67 \cdot 8$ & \\
\hline Caesarean section & 70 & $72 \cdot 2$ & 27 & $27 \cdot 8$ & 97 & 33.2 & \\
\hline \multicolumn{8}{|l|}{ Mothers } \\
\hline \multicolumn{7}{|l|}{ Age (years) } & 0.96 \\
\hline $20-30$ & 143 & 76.9 & 43 & $23 \cdot 1$ & 186 & $61 \cdot 8$ & \\
\hline$<20$ & 7 & $77 \cdot 8$ & 2 & $22 \cdot 2$ & 9 & 3.0 & \\
\hline$\geq 30$ & 80 & $75 \cdot 5$ & 26 & 24.5 & 106 & $35 \cdot 2$ & \\
\hline \multicolumn{7}{|l|}{ Education (years) } & 0.84 \\
\hline$\geq 12$ & 51 & 78.5 & 14 & 21.5 & 65 & $21 \cdot 6$ & \\
\hline$\overline{9}-12$ & 108 & $75 \cdot 0$ & 36 & $25 \cdot 0$ & 144 & 47.8 & \\
\hline$<9$ & 71 & $77 \cdot 2$ & 21 & $22 \cdot 8$ & 92 & $30 \cdot 6$ & \\
\hline \multicolumn{7}{|l|}{ Marital status } & 0.84 \\
\hline Marriage/consensual union & 206 & $76 \cdot 6$ & 63 & 23.4 & 269 & 89.4 & \\
\hline Others & 24 & $75 \cdot 0$ & 8 & $25 \cdot 0$ & 32 & $10 \cdot 6$ & \\
\hline Monthly household income (MS)† & & & & & & & 0.53 \\
\hline$\geq 5$ & 41 & $75 \cdot 9$ & 13 & $24 \cdot 1$ & 54 & $18 \cdot 1$ & \\
\hline $3-5$ & 57 & 81.4 & 13 & 18.6 & 70 & 23.5 & \\
\hline$<3$ & 130 & 74.7 & 44 & $25 \cdot 3$ & 174 & 58.4 & \\
\hline Working/studying after delivery* & & & & & & & 0.78 \\
\hline No & 89 & $75 \cdot 4$ & 29 & 24.6 & 118 & 39.5 & \\
\hline Yes & 139 & $76 \cdot 8$ & 42 & $23 \cdot 2$ & 181 & 60.5 & \\
\hline Smoking after pregnancy & & & & & & & 0.67 \\
\hline No & 202 & $76 \cdot 8$ & 61 & $23 \cdot 2$ & 263 & 87.4 & \\
\hline Yes & 28 & 73.7 & 10 & $26 \cdot 3$ & 38 & $12 \cdot 6$ & \\
\hline Pre-pregnancy BMI $\left(\mathrm{kg} / \mathrm{m}^{2}\right)$ & & & & & & & $<0.01$ \\
\hline$<25.0$ & 164 & $87 \cdot 7$ & 23 & $12 \cdot 3$ & 187 & $62 \cdot 2$ & \\
\hline $25 \cdot 0-30 \cdot 0$ & 46 & 60.5 & 30 & 39.5 & 76 & $25 \cdot 2$ & \\
\hline$\geq 30 \cdot 0$ & 20 & $52 \cdot 6$ & 18 & 47.4 & 38 & $12 \cdot 6$ & \\
\hline Gestational weight gain & & & & & & & $<0.01$ \\
\hline Adequate & 141 & 86.5 & 22 & 13.5 & 163 & $54 \cdot 2$ & \\
\hline Excessive & 89 & 64.5 & 49 & 35.5 & 138 & $45 \cdot 8$ & \\
\hline Pregnancies $(n)$ & & & & & & & 0.25 \\
\hline 2 & 84 & $81 \cdot 6$ & 19 & 18.4 & 103 & 34.2 & \\
\hline 3 & 60 & 71.4 & 24 & 28.6 & 84 & $27 \cdot 9$ & \\
\hline$\geq 4$ & 86 & $75 \cdot 4$ & 28 & $24 \cdot 6$ & 114 & 37.9 & \\
\hline
\end{tabular}

SGA, small for gestational age; AGA, adequate for gestational age; LGA, large for gestational age; MS, minimum salary (\$US 306.00).

${ }^{*} n 299$, two mothers did not know the breast-feeding duration, two mothers did not know the type of breast-feeding and two mothers did not know when they started working/studying after delivery.

† $n$ 298, three mothers did not know the monthly household income.

the future body weight of both mother and child will inform health professionals who have contact with women during their pregnancies and in the postpartum period about the importance of encouraging mothers to continue to breast-feed for at least the first 6 months of their child's life.
According to Gillman et $a l^{(14)}$, there are at least two mechanisms that may explain how longer breast-feeding duration protects infants against being overweight later in life. The first is behavioural and is related to children's own natural control of energy intake ${ }^{(14)}$. 
Table 3 Determinants of excess body weight at two years postpartum among Brazilian mothers ( $n$ 301) and their children ( $n$ 305), Joinville, Brazil, 2013-2014

\begin{tabular}{|c|c|c|c|c|c|c|c|c|c|c|c|c|c|c|c|c|c|c|}
\hline \multirow[b]{3}{*}{ Characteristic } & \multicolumn{6}{|c|}{ Children ( $n$ 305) } & \multicolumn{6}{|c|}{ Mothers ( $n$ 301) } & \multicolumn{6}{|c|}{ Mother-child pairs ( $n$ 71) } \\
\hline & \multicolumn{3}{|c|}{ Model $1^{*}$} & \multicolumn{3}{|c|}{ Model $2 \dagger$} & \multicolumn{3}{|c|}{ Model $3^{*}$} & \multicolumn{3}{|c|}{ Model 4† } & \multicolumn{3}{|c|}{ Model $5^{*}$} & \multicolumn{3}{|c|}{ Model $6 \dagger$} \\
\hline & OR & $95 \% \mathrm{Cl}$ & $P$ value & OR & $95 \% \mathrm{Cl}$ & $P$ value & OR & $95 \% \mathrm{Cl}$ & $P$ value & OR & $95 \% \mathrm{Cl}$ & $P$ value & OR & $95 \% \mathrm{Cl}$ & $P$ value & OR & $95 \% \mathrm{Cl}$ & $P$ value \\
\hline \multicolumn{19}{|c|}{ Breast-feeding duration (months) } \\
\hline$\geq 6$ & & ference & & $\operatorname{Ref}$ & ference & & & ference & & & ference & & & ference & & & ference & \\
\hline$\overline{4}-<6$ & 1.3 & $0.7,2.3$ & 0.40 & 1.4 & $0.7,2.6$ & 0.33 & $1 \cdot 7$ & $0.9,2.9$ & 0.07 & $2 \cdot 1$ & $0.9,5.1$ & 0.08 & $1 \cdot 1$ & $0.5,2 \cdot 2$ & 0.88 & 0.9 & $0.4,2.0$ & 0.71 \\
\hline $2-<4$ & $2 \cdot 0$ & $1 \cdot 0,4 \cdot 1$ & 0.04 & 1.8 & $0.8,3.7$ & 0.15 & $2 \cdot 1$ & $1 \cdot 0,4 \cdot 2$ & 0.03 & 2.5 & $0.9,7.2$ & 0.08 & $2 \cdot 3$ & $1 \cdot 0,5 \cdot 0$ & 0.04 & 1.7 & $0.6,4.3$ & 0.30 \\
\hline$<2$ & 2.7 & $1.5,7 \cdot 0$ & $<0.01$ & $2 \cdot 4$ & $1 \cdot 1,5 \cdot 1$ & 0.02 & 1.5 & $0.8,2.9$ & 0.24 & 2.9 & $1 \cdot 1,8 \cdot 1$ & 0.04 & $2 \cdot 6$ & $1 \cdot 2,5 \cdot 5$ & 0.01 & $3 \cdot 2$ & $1 \cdot 3,8 \cdot 2$ & 0.01 \\
\hline \multicolumn{19}{|l|}{ Months since delivery } \\
\hline$<18$ & \multicolumn{2}{|c|}{ Reference } & \multicolumn{4}{|c|}{ Reference } & \multicolumn{2}{|c|}{ Reference } & \multicolumn{4}{|c|}{ Reference } & \multicolumn{2}{|c|}{ Reference } & \multicolumn{4}{|c|}{ Reference } \\
\hline$\geq 18$ & 1.5 & $0.9,2.5$ & 0.07 & 1.7 & $1 \cdot 0,2 \cdot 9$ & 0.05 & 1.6 & $1 \cdot 0,2 \cdot 6$ & 0.04 & 1.8 & $0.9,3.8$ & 0.11 & $1 \cdot 8$ & $1 \cdot 0,3 \cdot 0$ & 0.03 & $1 \cdot 8$ & $0.9,3.5$ & 0.09 \\
\hline Sex & & & & & & & & & & & & & & & & & & \\
\hline Male & & ference & & Ref & ference & & & ference & & & ference & & $\operatorname{Re}$ & ference & & & ference & \\
\hline Female & 0.9 & $0.6,1.5$ & 0.82 & 0.9 & $0.6,1.5$ & 0.79 & 0.9 & $0.6,1.5$ & 0.76 & 1.0 & $0.5,1.9$ & 0.88 & 1.0 & $0.6,1.7$ & 0.98 & 1.0 & $0.5,1.8$ & 0.88 \\
\hline Birth weight & & & & & & & & & & & & & & & & & & \\
\hline SGA/AGA & & ference & & Ref & ference & & & ference & & & ference & & $\operatorname{Re}$ & ference & & & ference & \\
\hline LGA & $2 \cdot 4$ & $1.4,4.0$ & $<0.01$ & $2 \cdot 7$ & $1.5,4.9$ & $<0.01$ & 1.6 & $1 \cdot 0,2 \cdot 7$ & 0.07 & 1.5 & $0.7,3.3$ & 0.33 & 2.9 & $1 \cdot 6,5 \cdot 0$ & $<0.01$ & $3 \cdot 3$ & $1 \cdot 6,6 \cdot 6$ & $<0.01$ \\
\hline Type of delivery & & & & & & & & & & & & & & & & & & \\
\hline Normal & & ference & & Ref & ference & & & ference & & & ference & & $\operatorname{Re}$ & ference & & & ference & \\
\hline Caesarean section & $1 \cdot 2$ & $0.7,1.9$ & 0.53 & $1 \cdot 1$ & $0.6,1.9$ & $0 \cdot 77$ & 1.3 & $0 \cdot 8,2 \cdot 1$ & 0.25 & 1.0 & $0.5,2 \cdot 3$ & 0.94 & 1.4 & $0.8,2 \cdot 4$ & 0.23 & $1 \cdot 1$ & $0.5,2 \cdot 3$ & 0.78 \\
\hline Mother's age (years) & & & & & & & & & & & & & & & & & & \\
\hline $\begin{array}{l}<30 \\
\geq 30\end{array}$ & $\begin{array}{r}\mathrm{Re} \\
1.0\end{array}$ & $\begin{array}{l}\text { ference } \\
0.6,1.6\end{array}$ & 0.95 & $\begin{array}{c}\text { Ref } \\
1.1\end{array}$ & $\begin{array}{l}\text { ference } \\
0.6,2 \cdot 1\end{array}$ & 0.71 & $\begin{array}{rl}R & R \\
1.5\end{array}$ & $\begin{array}{l}\text { ference } \\
0.9,2.5\end{array}$ & 0.08 & $\begin{array}{l}R \in \\
0.7\end{array}$ & $\begin{array}{l}\text { ference } \\
0.3,1.7\end{array}$ & 0.46 & $\begin{aligned} \operatorname{Re} \\
1.1\end{aligned}$ & $\begin{array}{l}\text { ference } \\
0.6,1.9\end{array}$ & 0.77 & $\begin{array}{l}\mathrm{Re} \\
0.8\end{array}$ & $\begin{array}{l}\text { ference } \\
0.4,1.8\end{array}$ & 0.62 \\
\hline Mother's education (years & & & & & & & & & & & & & & & & & & \\
\hline$\geq 12$ & & ference & & & ference & & & ference & & & ference & & & ference & & & ference & \\
\hline$\overline{9}-12$ & & $0.8,2.5$ & 0.30 & 1.3 & $0.7,2.5$ & 0.46 & 0.9 & $0.5,1.5$ & 0.59 & 0.6 & $0.2,1.5$ & 0.26 & & $0.6,2.5$ & 0.58 & $1 \cdot 1$ & $0.5,2 \cdot 7$ & 0.79 \\
\hline$<9$ & $1 \cdot 2$ & $0 \cdot 6,2 \cdot 3$ & 0.56 & $1 \cdot 2$ & $0.6,2.5$ & 0.65 & 1.5 & $0.8,2 \cdot 8$ & 0.22 & 1.5 & $0.5,4.1$ & 0.42 & $1 \cdot 1$ & $0.5,2 \cdot 3$ & 0.84 & $1 \cdot 0$ & $0.4,2 \cdot 6$ & 0.93 \\
\hline Marital status & & & & & & & & & & & & & & & & & & \\
\hline Married/living together & & ference & & Ref & ference & & & ference & & & ference & & $\operatorname{Re}$ & ference & & $\mathrm{Re}$ & ference & \\
\hline Other & $1 \cdot 1$ & $0.5,2 \cdot 2$ & 0.89 & 1.0 & $0.4,2.5$ & 0.92 & 0.6 & $0.3,1.3$ & 0.17 & 0.8 & $0.2,2 \cdot 7$ & 0.73 & $1 \cdot 1$ & $0.5,2.5$ & 0.84 & 1.4 & $0.4,4.6$ & 0.59 \\
\hline Monthly household incom & e $(M S$ & & & & & & & & & & & & & & & & & \\
\hline$\geq 3$ & & ference & & Ref & ference & & & ference & & & ference & & $\operatorname{Re}$ & ference & & & ference & \\
\hline$<3$ & 1.5 & $0.9,2.4$ & 0.08 & 1.8 & $1 \cdot 0,3 \cdot 1$ & 0.03 & 1.3 & $0.8,2 \cdot 1$ & 0.23 & 1.0 & $0.5,2.0$ & 0.96 & 1.3 & $0.8,2 \cdot 3$ & 0.33 & 1.3 & $0.6,2.6$ & 0.49 \\
\hline Smoking after pregnancy & & & & & & & & & & & & & & & & & & \\
\hline No & & ference & & Ref & ference & & & ference & & & ference & & $\operatorname{Re}$ & ference & & $\mathrm{Re}$ & ference & \\
\hline Yes & 0.8 & $0.4,1.6$ & 0.46 & 0.9 & $0.4,1.9$ & 0.68 & $1 \cdot 3$ & $0.7,2 \cdot 7$ & 0.39 & 0.9 & $0.3,2 \cdot 6$ & 0.90 & $1 \cdot 2$ & $0.5,2 \cdot 6$ & 0.67 & 1.4 & $0.5,3.7$ & 0.48 \\
\hline Pre-pregnancy BMI $(\mathrm{kg} / \mathrm{m}$ & & & & & & & & & & & & & & & & & & \\
\hline$<25.0$ & & ference & & & ference & & & ference & & & ference & & & ference & & & ference & \\
\hline $\begin{array}{l}\geq 25 \cdot 0 \\
\text { GWG }\end{array}$ & $1 \cdot 2$ & $0 \cdot 7,1 \cdot 8$ & 0.55 & $1 \cdot 0$ & $0.6,1 \cdot 7$ & 0.86 & $30 \cdot 4$ & $15 \cdot 0,61 \cdot 7$ & $<0.01$ & $30 \cdot 9$ & $13 \cdot 6,70 \cdot 2$ & $<0.01$ & $5 \cdot 2$ & $2 \cdot 9,9 \cdot 2$ & $<0.01$ & $5 \cdot 7$ & $2 \cdot 8,11 \cdot 8$ & $<0.01$ \\
\hline Adequate & & ference & & & ference & & & ference & & & ference & & & ference & & & ference & \\
\hline $\begin{array}{l}\text { Excessive } \\
\text { Pregnancies }(n)\end{array}$ & 1.6 & $1 \cdot 0,2 \cdot 5$ & 0.04 & 1.5 & $0.9,2.5$ & 0.14 & 4.4 & $2 \cdot 7,7 \cdot 2$ & $<0.01$ & 3.4 & $1 \cdot 7,6.9$ & $<0.01$ & 3.5 & $2 \cdot 0,6 \cdot 2$ & $<0.01$ & $2 \cdot 2$ & $1 \cdot 1,4 \cdot 3$ & 0.02 \\
\hline 2 & & ference & & & ference & & & ference & & & ference & & & ference & & & ference & \\
\hline 3 & $1 \cdot 1$ & $0.6,2.0$ & 0.75 & 0.9 & $0.4,1.4$ & 0.78 & 2.6 & $1.4,4.7$ & $<0.01$ & 1.2 & $0.5,3.0$ & 0.75 & & $0.9,3.5$ & $0 \cdot 10$ & & $0.4,2.6$ & 0.90 \\
\hline$\geq 4$ & 1.0 & $0.6,1 \cdot 7$ & 0.96 & 0.7 & $0.3,1.4$ & 0.28 & $3 \cdot 1$ & $1.8,5.5$ & $<0.01$ & $2 \cdot 8$ & $1 \cdot 1,7 \cdot 2$ & 0.03 & 1.4 & $0.7,2 \cdot 8$ & 0.27 & 0.9 & $0.4,2 \cdot 4$ & 0.86 \\
\hline
\end{tabular}


(a)

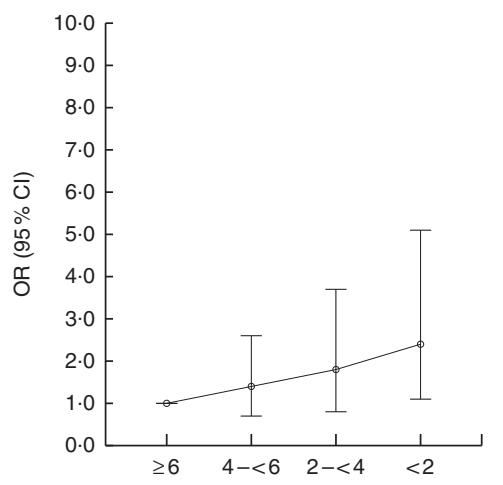

(b)

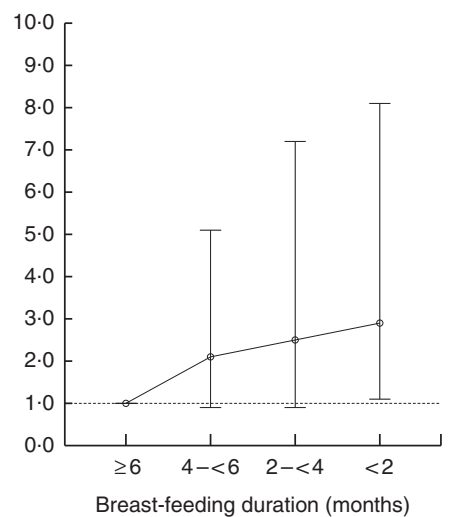

(c)

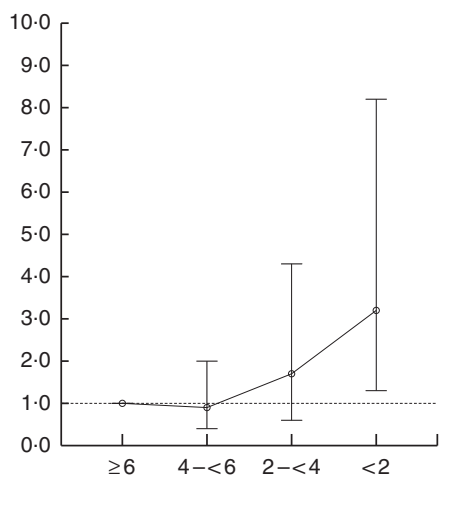

Fig. 2 Risk of excess body weight in (a) children ( $n$ 305), (b) mothers ( $n$ 301) and (c) mother-child pairs $(n 71)$ by breast-feeding duration, Joinville, Brazil, 2013-2014. Values are odds ratios with their $95 \%$ confidence intervals represented by vertical bars; - . - - - represents the null effect

Parents' behaviours that influence their child's eating habits may override appetite signals ${ }^{(14)}$, eventually contributing to the modification of a child's natural 'selfcontrol'. The second suggested mechanism involves the metabolic consequences of ingesting breast milk $^{(14)}$, which is more energy-dense, more easily metabolized and consumed in smaller amounts compared with formula ${ }^{(53)}$.

Although it is often suggested that the relationship between breast-feeding practices and maternal postpartum weight loss is due to the increased energy demands of breast-feeding, other possible mechanisms may play an important role. For example, high leptin and insulin levels present in mothers at delivery may be one of several possible contributing factors responsible for this increased body weight in the postpartum period in mothers who do not breast-feed. Schubring et al. ${ }^{(54)}$ suggested that during late pregnancy and at birth, serum leptin levels are high to help increase energy stores required during delivery and lactation after birth. Serum leptin levels decrease throughout lactation; therefore, mothers who do not breast-feed continue to have higher leptin levels for a longer period of time, which may contribute to an increased body weight compared with those who breast-feed. In addition, elevated leptin and insulin levels in the postpartum period can lead to leptin and insulin resistance, impairing appetite regulation and increasing the risk of obesity ${ }^{(55)}$. Appetite-regulating hormones like leptin and insulin might also influence infant body composition. Breij et al. ${ }^{(56)}$ found higher leptin and insulin levels in infants fed formula for at least 3 months compared with breast-feeding, and both hormones were positively correlated with fat mass percentage.

Presently, many countries around the world, especially emerging economies such as Brazil, are experiencing a nutrition transition ${ }^{(57)}$. Urbanization and shifts in dietary patterns and levels of physical activity in these countries are fuelling the increased rates of overweight and obesity ${ }^{(57,58)}$. Therefore, to reduce the global burden of chronic disease, public health strategies focused on primary prevention of obesity early in life and at critical periods throughout the life span, such as during pregnancy and the postpartum period, should be considered.

Early infancy up to age 2 years is a crucial period in a child's development as many obesity-related lifestyle habits are formed at this early stage and unhealthy habits are likely to continue throughout childhood and later in life $\mathrm{f}^{(10,11,59)}$. Parents play a critical role in their child's development and make important decisions that affect all aspects of their child's life, including what they eat and consequently their body weight ${ }^{(59)}$. Therefore, it is important to involve parents in prevention and intervention strategies that target children.

In terms of public health, it is clear that comprehensive efforts should be directed at mothers during the first months after birth so that breast-feeding is indeed acquired. Although mothers attend the prenatal visits, sometimes abandoning or not breast-feeding occurs due to inexperience or even cultural issues. Such features may be circumvented with longer and more effective follow-up after delivery, either through a greater number of paediatrician visits or weekly home visits by health-care professionals.

A major strength of the current study was the analyses of excess body weight concurrently in mother-child pairs, as this type of analysis has not been performed in previous studies. Prospective data collection and the ability to adjust for several important confounding factors are also important strengths of the study. Finally, the same researchers performed data collection, including anthropometric measurements, at baseline and follow-up, therefore reducing possible biases.

A limitation of the present study was the inclusion of self-reported variables, including pre-pregnancy weight, smoking, household income and breast-feeding duration, as such variables are vulnerable to reporter biases. Second, our results are from a relatively small cohort of mothers and their children living in Brazil; therefore, caution should be taken when the results are compared with other 
populations. Although the children in the study were followed only for 2 years, the association between breastfeeding duration and weight status at this age increases our understanding of the impact of breast-feeding during infancy and provides insight into the risk of excess body weight later in life ${ }^{(23)}$. Lastly, as with most longitudinal studies, a degree of measurement error may have occurred that could have affected the accuracy of both prepartum and postpartum variables used in the study ${ }^{(28)}$.

\section{Conclusions}

Unlike previously performed studies, the current investigation concurrently examined the effects of breast-feeding duration on the body weight of mother-child pairs. Findings from the study indicated that breast-feeding for a longer duration has a protective effect on the risk of excess body weight concurrently in both mothers and their children two years after birth. These results are important from a public health perspective as strategies aimed at promoting longer breast-feeding duration could help to attenuate the rising prevalence of overweight and obesity for both mother and child.

\section{Acknowledgements}

Acknowledgements: The authors thank Darcy Vargas Maternity Hospital and the Gimenes Laboratory of Joinville, Santa Catarina, Brazil for allowing data to be collected from their facilities, the University of Joinville Region for its financial support, and the Population Health Intervention Research Unit/School of Public Health/University of Alberta, Canada for its statistical and financial support. Financial support: This study was funded by research grants from the University of Joinville Region, Joinville, Santa Catarina, Brazil (grant number 4555/2011). M.F.M. was supported by the National Council for Scientific and Technological Development, Brazil (grant number 249048/2013-2) and by the Population Health Intervention Research Unit (PHIRU), School of Public Health, University of Alberta, Alberta, Canada with a postdoctoral fellowship. S.S.B.S.M. was also supported by PHIRU with a postdoctoral fellowship. The funders had no role in the design, analysis or writing of this article. Conflict of interest: The authors declare that they have no conflicts of interest. Authorship: M.F.M. conceptualized and designed the study, drafted the initial manuscript and analyses, coordinated and supervised data collection. S.S.B.S.M. contributed to design the study, designed the data collection instruments and revised the manuscript. S.A.C contributed to the data collection and revised the manuscript. J.P.E. contributed to the statistical analysis and revised the manuscript. S.A.L. drafted and revised the manuscript. P.J.V. contributed to the statistical analysis, drafted and revised the manuscript. All authors read and approved the final manuscript. Ethics of buman subject participation: This study was conducted according to the guidelines laid down in the Declaration of Helsinki and all procedures involving human subjects/patients were approved by the research ethics committee of the University of Joinville Region (number 107/2011). All participants gave their written and informed consent.

\section{References}

1. Kirkegaard H, Stovring H, Rasmussen KM et al. (2014) How do pregnancy-related weight changes and breastfeeding relate to maternal weight and BMI-adjusted waist circumference $7 \mathrm{y}$ after delivery? Results from a path analysis. Am J Clin Nutr 99, 312-319.

2. Instituto Brasileiro de Geografia e Estatística (2010) Pesquisa de Orçamentos Familiares 2008-2009. Antropometria e Estado Nutricional de Crianças, Adolescentes e Adultos no Brasil. Rio de Janeiro: IGBE.

3. He X, Zhu M, Hu C et al. (2015) Breast-feeding and postpartum weight retention: a systematic review and metaanalysis. Public Health Nutr 18, 3308-3316.

4. Rooney BL \& Schauberger CW (2002) Excess pregnancy weight gain and long-term obesity: one decade later. Obstet Gynecol 100, 245-252.

5. Linne Y \& Neovius M (2006) Identification of women at risk of adverse weight development following pregnancy. Int J Obes (Lond) 30, 1234-1239.

6. Stocks T, Renders CM, Bulk-Bunschoten AM et al. (2011) Body size and growth in 0- to 4-year-old children and the relation to body size in primary school age. Obes Rev 12, 637-652.

7. Gardner DS, Hosking J, Metcalf BS et al. (2009) Contribution of early weight gain to childhood overweight and metabolic health: a longitudinal study (EarlyBird 36). Pediatrics 123, e67-e73.

8. Stettler N \& Iotova V (2010) Early growth patterns and long-term obesity risk. Curr Opin Clin Nutr Metab Care 13, 294-299.

9. Kaar JL, Crume T, Brinton JT et al. (2014) Maternal obesity, gestational weight gain, and offspring adiposity: the exploring perinatal outcomes among children study. $J$ Pediatr 165, 509-515.

10. Brown A \& Lee M (2012) Breastfeeding during the first year promotes satiety responsiveness in children aged 18-24 months. Pedriatr Obes 7, 382-390.

11. Gubbels JS, Thijs C, Stafleu A et al. (2011) Association of breast-feeding and feeding on demand with child weight status up to 4 years. Int J Pediatr Obes 6, e515-e522.

12. Burke V, Beilin LJ, Simmer K et al. (2005) Breastfeeding and overweight: longitudinal analysis in an Australian birth cohort. J Pediatr 147, 56-61.

13. Carling SJ, Demment MM, Kjolhede CL et al. (2015) Breastfeeding duration and weight gain trajectory in infancy. Pediatrics 135, 111-119.

14. Gillman MW, Rifas-Shiman SL, Camargo CA Jr et al. (2001) Risk of overweight among adolescents who were breastfed as infants. JAMA 285, 2461-2467.

15. Gillman MW, Rifas-Shiman SL, Kleinman K et al. (2008) Developmental origins of childhood overweight: potential public health impact. Obesity (Silver Spring) 16, 1651-1656.

16. Gunnarsdottir I, Schack-Nielsen L, Michaelsen KF et al. (2010) Infant weight gain, duration of exclusive breastfeeding and childhood BMI - two similar follow-up cohorts. Public Health Nutr 13, 201-207. 
17. Hunsberger M, Lanfer A, Reeske A et al. (2013) Infant feeding practices and prevalence of obesity in eight European countries - the IDEFICS study. Public Health Nutr 16, 219-227.

18. Huus K, Ludvigsson JF, Enskar K et al. (2008) Exclusive breastfeeding of Swedish children and its possible influence on the development of obesity: a prospective cohort study. BMC Pediatr 8, 42.

19. McCrory C \& Layte R (2012) Breastfeeding and risk of overweight and obesity at nine-years of age. Soc Sci Med 75, 323-330.

20. Nelson MC, Gordon-Larsen P \& Adair LS (2005) Are adolescents who were breast-fed less likely to be overweight? Analyses of sibling pairs to reduce confounding. Epidemiology 16, 247-253.

21. Taveras EM, Rifas-Shiman SL, Scanlon KS et al. (2006) To what extent is the protective effect of breastfeeding on future overweight explained by decreased maternal feeding restriction? Pediatrics 118, 2341-2348.

22. Weyermann M, Rothenbacher D \& Brenner H (2006) Duration of breastfeeding and risk of overweight in childhood: a prospective birth cohort study from Germany. Int J Obes (Lond) 30, 1281-1287.

23. Zheng JS, Liu H, Li J et al. (2014) Exclusive breastfeeding is inversely associated with risk of childhood overweight in a large Chinese cohort. J Nutr 144, 1454-1459.

24. Wen LM, Baur LA, Rissel C et al. (2014) Correlates of body mass index and overweight and obesity of children aged 2 years: findings from the healthy beginnings trial. Obesity (Silver Spring) 22, 1723-1730.

25. Donath SM \& Amir LH (2008) Maternal obesity and initiation and duration of breastfeeding: data from the longitudinal study of Australian children. Matern Child Nutr 4, 163-170.

26. Endres LK, Straub H, McKinney C et al. (2015) Postpartum weight retention risk factors and relationship to obesity at 1 year. Obstet Gynecol 125, 144-152.

27. Sichieri R, Field AE, Rich-Edwards J et al. (2003) Prospective assessment of exclusive breastfeeding in relation to weight change in women. Int J Obes Relat Metab Disord 27, 815-820.

28. Ng SK, Cameron CM, Hills AP et al. (2014) Socioeconomic disparities in prepregnancy BMI and impact on maternal and neonatal outcomes and postpartum weight retention: the EFHL longitudinal birth cohort study. BMC Pregnancy Childbirth 14, 314.

29. Bertotto ML, Valmórbida J, Broilo MC et al. (2012) Associação entre ganho de peso no primeiro ano de vida com excesso de peso e adiposidade abdominal na idade préescolar. Rev Paulista Pediatr 30, 507-512.

30. Araujo CL, Victora CG, Hallal PC et al. (2006) Breastfeeding and overweight in childhood: evidence from the Pelotas 1993 birth cohort study. Int J Obes (Lond) 30, 500-506.

31. Burdette HL, Whitaker RC, Hall WC et al. (2006) Breastfeeding, introduction of complementary foods, and adiposity at 5 y of age. Am J Clin Nutr $\mathbf{8 3}, 550-558$.

32. Caleyachetty A, Krishnaveni GV, Veena SR et al. (2013) Breastfeeding duration, age of starting solids and high BMI risk and adiposity in Indian children. Matern Child Nutr 9 , 199-216.

33. Demment MM, Haas JD \& Olson CM (2014) Changes in family income status and the development of overweight and obesity from 2 to 15 years: a longitudinal study. BMC Public Health 14, 417.

34. Durmus B, van Rossem L, Duijts L et al. (2011) Breastfeeding and growth in children until the age of 3 years: the Generation R Study. Br J Nutr 105, 1704-1711.

35. Kamudoni $\mathrm{P}$, Maleta $\mathrm{K}$, Shi $\mathrm{Z}$ et al. (2015) Exclusive breastfeeding duration during the first 6 months of life is positively associated with length-for-age among infants 6-12 months old, in Mangochi district, Malawi. Eur J Clin Nutr 69, 96-101.
36. Kwok MK, Schooling CM, Lam TH et al. (2010) Does breastfeeding protect against childhood overweight? Hong Kong's 'Children of 1997' birth cohort. Int J Epidemiol 39, 297-305.

37. Neutzling MB, Hallal PR, Araujo CL et al. (2009) Infant feeding and obesity at 11 years: prospective birth cohort study. Int J Pediatr Obes 4, 143-149.

38. Victora CG, Barros F, Lima RC et al. (2003) Anthropometry and body composition of 18 year old men according to duration of breast feeding: birth cohort study from Brazil. BMJ 327, 901.

39. Jiang M \& Foster EM (2013) Duration of breastfeeding and childhood obesity: a generalized propensity score approach. Health Serv Res 48, 628-651.

40. Lefebvre CM \& John RM (2014) The effect of breastfeeding on childhood overweight and obesity: a systematic review of the literature. J Am Assoc Nurse Pract 26, 386-401.

41. Chiasson MA, Scheinmann R, Hartel D et al. (2016) Predictors of obesity in a cohort of children enrolled in WIC as infants and retained to 3 years of age. J Community Health 41, 127-133.

42. Mastroeni MF, Czarnobay SA, Kroll C et al. (2017) The independent importance of pre-pregnancy weight and gestational weight gain for the prevention of large-for gestational age Brazilian newborns. Matern Child Health J 21, 705-714.

43. Sales WB, Silleno Junior JD, Kroll C et al. (2015) Influence of altered maternal lipid profile on the lipid profile of the newborn. Arch Endocrinol Metab 59, 123-128.

44. World Health Organization (2000) Obesity: Preventing and Managing the Global Epidemic. Report of a WHO Consultation. WHO Technical Report Series no. 894. Geneva: WHO.

45. Rasmussen KM \& Yaktine A (2009) Weight Gain During Pregnancy: Reexamining the Guidelines. Washington, DC: National Academies Press.

46. Lubchenco LO, Hansman C, Dressler M et al. (1963) Intrauterine growth as estimated from liveborn birth-weight data at 24 to 42 weeks of gestation. Pediatrics 32, 793-800.

47. De Onis M (2006) WHO Child Growth Standards: Length/ Height-for-Age, Weight-for-Age, Weight-for-Length, Weightfor-Height and Body Mass Index-for-Age: Methods and Development. Geneva: WHO.

48. World Health Organization (2008) Indicators for Assessing Infant and Young Child Feeding Practices: Part 1: Definitions. Conclusions of a consensus meeting beld 6-8 November 2007 in Washington, DC, USA. Geneva: WHO.

49. Instituto Brasileiro de Geografia e Estatística (2010) Pesquisa Nacional por Amostra de Domicílios. http://www.ibge.gov. br/home/estatistica/populacao/trabalhoerendimento/pnad98/ saude/analise.shtm (accessed April 2017).

50. Brasil (1996) Lei $n^{\circ}$ 9.394, de 20 de dezembro de 1996. Estabelece as Diretrizes e Bases da Educação Nacional. http://www.planalto.gov.br/ccivil_03/Leis/L9394.htm (accessed April 2017).

51. O'Brien R (2007) A caution regarding rules of thumb for variance inflation factors. Qual Quant 41, 673-690.

52. Gigante DP, Victora CG \& Barros FC (2001) Breast-feeding has a limited long-term effect on anthropometry and body composition of Brazilian mothers. J Nutr 131, 78-84.

53. Lucas A, Boyes S, Bloom SR et al. (1981) Metabolic and endocrine responses to a milk feed in six-day-old term infants: differences between breast and cow's milk formula feeding. Acta Paediatr Scand 70, 195-200.

54. Schubring C, Blum WF, Kratzsch J et al. (2000) Leptin, the ob gene product, in female health and disease. Eur J Obstet Gynecol Reprod Biol 88, 121-127.

55. Andreas NJ, Hyde MJ, Gale C et al. (2014) Effect of maternal body mass index on hormones in breast milk: a systematic review. PLoS One $\mathbf{9}$, e115043. 
56. Breij LM, Mulder MT, van Vark-van der Zee LC et al. (2016) Appetite-regulating hormones in early life and relationships with type of feeding and body composition in healthy term infants. Eur J Nutr (Epublication ahead of print version).

57. Conde WL \& Monteiro CA (2014) Nutrition transition and double burden of undernutrition and excess of weight in Brazil. Am J Clin Nutr 100, issue 6, 1617S-1622S.

58. Popkin BM (2001) The nutrition transition and obesity in the developing world. J Nutr 131, issue 3, 871S-873S.

59. Gubbels JS, Kremers SP, Stafleu A et al. (2009) Clustering of dietary intake and sedentary behavior in 2-year-old children. J Pediatr 155, 194-198. 